\title{
SOME APPLICATIONS OF OCCUPATION TIMES OF BROWNIAN MOTION WITH DRIFT IN MATHEMATICAL FINANCE
}

\author{
ANDREAS PECHTL \\ Center of Asset Pricing and Financial Products Development \\ Deutsche Genossenschaftsbank Frankfurt am Main \\ Am Platz der Republik, D-60325 \\ Frankfurt am Main, Germany
}

\begin{abstract}
In the last few years new types of path-dependent options called corridor options or range options have become well-known derivative instruments in European options markets. Since the payout profiles of those options are based on occupation times of the underlying security the purpose of this paper is to provide closed form pricing formulae of Black \& Scholes type for some significant representatives. Alternatively we demonstrate in this paper a relatively simple derivation of the Black \& Scholes price for a single corridor option - based on a static portfolio representation - which does not make use of the distribution of occupation times (of Brownian motion). However, knowledge of occupation times' distributions is a more powerful tool.
\end{abstract}

Keywords: Brownian motion with drift, occupation times, Black \& Scholes model, corridor options.

\section{INTRODUCTION AND DESCRIPTION OF THE PROBLEM}

Since 1973 when Black and Scholes presented their seminal paper concerning the pricing of financial securities [see Black \& Scholes (1973)], where the no- arbitrage price-process $X=\left(X_{t} ; t \geq 0\right)$ of the underlying security is described by geometric Brownian motion, questions of option pricing have stimulated both, economics and stochastic calculus [see Harrison \& Pliska (1981 ), Karatzas \& Shreve (1988), Duffie (1988), et al.].

Recent results have been inspired by so-called Asian options or average options and induced a rigorous study of random variables obtained by application of average functionals to the price-process. A famous problem in mathematical finance has arisen from the application of arithmetic averages to $X$. To discover an analytic representation of the corresponding distribution has turned out to be a resistant problem for years and has been denoted jestingly "a minor holy grail in mathematical finance" [see Hart \& Ross (1994)] till Yor (1992) found a remarkable result using Laplace transformation.

Another type of average options are quantile options first introduced by Miura (1992). The $\alpha$-quantile $M(\alpha, T)(z)$ of a measurable function $z$ on $[0 ; T]$ is defined 
by

$$
M(\alpha, T)(z)=\inf \left\{k \in g R \mid \int_{t=0}^{T} \mathbf{1}[z(t) \leq k] d t>\alpha T\right\} .
$$

Questions concerning the distribution of $M(\alpha, T)(X)$ are closely related to questions of distribution concerning occupation times of Brownian motion with drift $Z=\left(Z_{t}, t \geq 0\right)$, i.e. the random variable $\Gamma_{+}(T, k)(Z)=\int_{t=0}^{T} \mathbf{1}\left[Z_{t}>k\right] d t$. The distribution of $M(\alpha, T)(Z)$ was studied successfully by Akahori (1995) and Dassios (1995). Though Lévy's arc- sine law for occupation times of standard Brownian motion is a classical probabilistic result [see Billingsley (1968)] it seems that explicit formulae for the distributions of occupation times of Brownian motion with drift have not been available since both, Akahori and Dassios, only provide formulae for densities respectively integral versions for the options' prices.

However, there is a practical need and interest in such explicit solutions if available, especially when dealing with path-dependent options, where established approximative methods, e.g. numerical integration, Monte Carlo simulation or lattice approximation, often fail or - at least - consume enormous resources of computation time. Motivated by those practical needs we provide the explicit representation for the distribution of $\Gamma_{+}(T, k)(Z)$ [see Pechtl (1997), Theorem 1.1 and Theorem 3.1] which can be used not only for pricing quantile options, but also for some types of corridor options.

While quantile options seem to be highly sophisticated financial products nearly unknown to investors corridor options or range options were introduced to European options markets in 1994 and have become familiar derivative instruments. The payout profile of a traditional single corridor option is given by

$$
\Psi_{1}\left(\lambda, T, K_{1}, K_{2} ; z\right)=\lambda \int_{t=-T_{0}}^{T} \mathbf{1}\left[K_{1}<z(t) \leq K_{2}\right] d t
$$

with $T, T_{0} \geq 0, \lambda>0$ and $-\infty \leq K_{1}<K_{2} \leq \infty$, whereas the more complex structure of a dual corridor option is given by

$$
\begin{aligned}
\Psi_{2}\left(\lambda_{1}, \lambda_{2}, T, K_{1}, K_{2} ; z\right)= & \left(\lambda_{1} \int_{t=-T_{0}}^{T} \mathbf{1}\left[z(t) \in\left(K_{1} ; K_{2}\right]\right] d t\right. \\
& \left.-\lambda_{2} \int_{t=0}^{T} \mathbf{1}\left[z(t) \notin\left(K_{1} ; K_{2}\right]\right] d t\right)^{+}
\end{aligned}
$$

with $\lambda_{1}, \lambda_{2} \in g R$.

The aim of this paper is to provide a general pricing formula for dual switch options, i.e. dual corridor options with $K_{2}=\infty$, and related derivative securities in the well-known Black \& Scholes model.

The paper is structured as follows. In Section 2 the Black \& Scholes assumptions and the notations used in the paper are introduced briefly. The main results are 
presented in Section 3, in particular the explicit version for the Black \& Scholes price of a dual switch option and its derivation as an application of the distribution of occupation times of Brownian motion with drift. An alternative and in the considered special case of single corridor options rather elegant method of evaluation of the option's price based on static replication techniques is provided in Section 4.

\section{PRELIMINARY REMARKS AND NOTATIONS}

Throughout this paper we consider pricing of derivative securities in the Black \& Scholes model, where the risk-neutral price-process $X=\left(X_{t}, t \geq 0\right)$, i.e. the process which has to be used for the calculation of the option's price, can be represented by

$$
X_{t}=S_{0} \exp \left\{\sigma W_{t}+\mu_{B S} t\right\}, 0 \leq t \leq T,
$$

with a one-dimensional standard Brownian motion $W=\left(W_{t}, t \geq 0\right)$, the initial price $S_{0}$ of the underlying security, its volatility $\sigma, r(>1)$ defined by one plus the risk-free interest rate and the drift

$$
\mu_{B S}=\log r-\frac{1}{2} \sigma^{2}
$$

determined by the model.

As well known the payout profile of an option is described by a real-valued functional $\Phi$ on the measurable space $\left(C[0 ; T] ; \mathcal{C}^{[0 ; T]}\right)$, the linear space of all continuous functions on the time-interval $[0 ; T]$ endowed with the $\sigma$-algebra of Borel sets. Then the Black \& Scholes price $\pi_{B S}(\Phi)$ is calculated by

$$
\pi_{B S}(\Phi)=r^{-T} E \Phi(X) .
$$

For the following explicit calculations we introduce the univariate and the bivariate standard normal distribution functions by

$$
\mathcal{N}(x)=\frac{1}{\sqrt{2 \pi}} \int_{v=-\infty}^{x} \exp \left\{-\frac{v^{2}}{2}\right\} d v
$$

for all $x \in g R$ and by

$\mathcal{N}(x, y ; \rho)=\frac{1}{2 \pi \sqrt{1-\rho^{2}}} \int_{v=-\infty}^{x} \int_{w=-\infty}^{y} \exp \left\{-\frac{1}{2\left(1-\rho^{2}\right)}\left(v^{2}-2 \rho v w+w^{2}\right)\right\} d w d v$ for all $x, y \in g R$ and all $\rho \in(-1 ; 1)$.

\section{THE PRICE OF A DUAL SWITCH OPTION}

For our purposes we introduce the Brownian motion with drift $Y=\left(Y_{t} ; t \geq 0\right)$ defined by

$$
Y_{t}=W_{t}+\frac{\mu_{B S}}{\sigma} t
$$


Furthermore let $\mathbf{F}$ the distribution function of the random variable $\Gamma_{+}(T, K)(X)$ with

$$
\begin{aligned}
\Gamma_{+}(T, K)(X) & =\int_{t=0}^{T} \mathbf{1}\left[X_{t}>K\right] d t=\int_{t=0}^{T} \mathbf{1}\left[Y_{t}>\frac{1}{\sigma} \log \frac{K}{S_{0}}\right] d t \\
& =\Gamma_{+}\left(T, \frac{1}{\sigma} \log \frac{K}{S_{0}}\right)(Y)
\end{aligned}
$$

i.e. $\mathbf{F}(t)=P\left[\Gamma_{+}\left(T, \frac{1}{\sigma} \log \frac{K}{S_{0}}\right)(Y) \leq t\right]$. By Theorem 1.1 and Theorem 3.1 in Pechtl (1997) $\mathbf{F}$ is explicitly known and can be represented in the following way,

$$
\begin{aligned}
& P\left[\Gamma_{+}\left(T, \frac{1}{\sigma} \log \frac{K}{S_{0}}\right)(Y) \leq t\right]=F\left(T, t, \frac{1}{\sigma} \log \frac{K}{S_{0}} ; \frac{\mu_{B S}}{\sigma}\right), K \leq S_{0} ; \\
& P\left[\Gamma_{+}\left(T, \frac{1}{\sigma} \log \frac{K}{S_{0}}\right)(Y) \leq t\right]=1-F\left(T, T-t, \frac{1}{\sigma} \log \frac{S_{0}}{K} ;-\frac{\mu_{B S}}{\sigma}\right), K>S_{0},
\end{aligned}
$$

where the function $F(\tau, \theta, \kappa ; \mu)$ is defined for all $\kappa<0$ and all $0 \leq \theta<\tau$ by

$$
\begin{aligned}
F(\tau, \theta, \kappa ; \mu)= & \left\{3+2 \kappa \mu+2 \mu^{2} \tau\right\} \exp \{2 \kappa \mu\} \\
& \mathcal{N}\left(\frac{\kappa}{\sqrt{\tau}}+\mu \sqrt{\tau},-\mu \sqrt{\tau-\theta} ;-\sqrt{1-\frac{\theta}{\tau}}\right) \\
& +\mathcal{N}\left(\frac{\kappa}{\sqrt{\tau}}-\mu \sqrt{\tau}, \mu \sqrt{\tau-\theta} ;-\sqrt{1-\frac{\theta}{\tau}}\right) \\
& -\left\{1+2 \kappa \mu+2 \mu^{2} \theta\right\} \exp \{2 \kappa \mu\} \mathcal{N}\left(\frac{\kappa}{\sqrt{\theta}}+\mu \sqrt{\theta}\right) \mathcal{N}(-\mu \sqrt{\tau-\theta}) \\
& +\mathcal{N}\left(\frac{\kappa}{\sqrt{\theta}}-\mu \sqrt{\theta}\right) \mathcal{N}(-\mu \sqrt{\tau-\theta}) \\
& -2 \mu \sqrt{\frac{\theta}{2 \pi}} \exp \left\{-\frac{1}{2}\left(\frac{\kappa}{\sqrt{\theta}}-\mu \sqrt{\theta}\right)^{2}\right\} \mathcal{N}(-\mu \sqrt{\tau-\theta}) \\
& +2 \mu \sqrt{\frac{\tau}{2 \pi}} \exp \left\{-\frac{1}{2}\left(\frac{\kappa}{\sqrt{\tau}}-\mu \sqrt{\tau}\right)^{2}\right\} \mathcal{N}\left(\kappa \sqrt{\frac{1}{\theta}-\frac{1}{\tau}}\right) \\
& -2 \mu \sqrt{\frac{\tau-\theta}{2 \pi}} \exp \left\{-\frac{1}{2} \mu^{2}(\tau-\theta)\right\} \exp \{2 \kappa \mu\} \mathcal{N}\left(\frac{\kappa}{\sqrt{\theta}}+\mu \sqrt{\theta}\right)
\end{aligned}
$$

For $\theta<0$ let be $F(\tau, \theta, \kappa ; \mu) \equiv 0$ and for $\theta \geq \tau$ let be $F(\tau, \theta, \kappa ; \mu) \equiv 1$. 
Furthermore we define for $\kappa<0$ the integral $J(\tau, \theta, \kappa ; \mu)=\int_{v=-\infty}^{\theta} F(\tau, v, \kappa ; \mu) d v$ and calculate

$$
\begin{array}{r}
J(\tau, \theta, \kappa ; \mu)=\left\{3+2 \kappa \mu+2 \mu^{2} \tau\right\} \theta \exp \{2 \kappa \mu\} \mathcal{N}\left(\frac{\kappa}{\sqrt{\tau}}+\mu \sqrt{\tau},-\mu \sqrt{\tau-\theta} ;-\sqrt{1-\frac{\theta}{\tau}}\right) \\
+\left\{\frac{1}{2 \mu^{2}}-\mu^{2} \tau^{2}-2 \tau+\kappa^{2}\right\} \exp \{2 \kappa \mu\} \mathcal{N}\left(\frac{\kappa}{\sqrt{\tau}}+\mu \sqrt{\tau},-\mu \sqrt{\tau-\theta} ;-\sqrt{1-\frac{\theta}{\tau}}\right) \\
+\left\{\theta-\frac{1}{2 \mu^{2}}-\frac{\kappa}{\mu}\right\} \mathcal{N}\left(\frac{\kappa}{\sqrt{\tau}}-\mu \sqrt{\tau}, \mu \sqrt{\tau-\theta} ;-\sqrt{1-\frac{\theta}{\tau}}\right) \\
-\left\{\mu^{2} \theta^{2}+2 \kappa \mu \theta+\theta+\kappa^{2}-\frac{1}{2 \mu^{2}}\right\} \exp \{2 \kappa \mu\} \mathcal{N}\left(\frac{\kappa}{\sqrt{\theta}}+\mu \sqrt{\theta}\right) \mathcal{N}(-\mu \sqrt{\tau-\theta}) \\
+\left\{\theta-\frac{\kappa}{\mu}-\frac{1}{2 \mu^{2}}\right\} \mathcal{N}\left(\frac{\kappa}{\sqrt{\theta}}-\mu \sqrt{\theta}\right) \mathcal{N}(-\mu \sqrt{\tau-\theta})(9) \\
+\left\{2 \mu \theta-\mu \tau-\frac{1}{\mu}+\kappa\right\} \sqrt{\frac{\tau}{2 \pi}} \exp \left\{-\frac{1}{2}\left(\frac{\kappa}{\sqrt{\tau}}-\mu \sqrt{\tau}\right)^{2}\right\} \mathcal{N}\left(\kappa \sqrt{\frac{1}{\theta}-\frac{1}{\tau}}\right) \\
\left.+\left\{\mu(\tau-\theta)+\frac{1}{\mu}\right\} \sqrt{\frac{\theta-\theta}{2 \pi}} \exp \{2 \kappa \mu\} \exp \left\{-\frac{1}{2}\left(\frac{\kappa}{\sqrt{\theta}}-\mu \sqrt{\theta}\right)^{2}\right\} \mathcal{N}(-\mu \sqrt{\tau-\theta})\right\} \mathcal{N}\left(\frac{\kappa}{\sqrt{\theta}}+\mu \sqrt{\theta}\right) \\
+\frac{\sqrt{\theta(\tau-\theta)}}{2 \pi} \exp \left\{-\frac{\mu^{2}}{2}(\tau-\theta)\right\} \exp \left\{-\frac{1}{2}\left(\frac{\kappa}{\sqrt{\theta}}-\mu \sqrt{\theta}\right)^{2}\right\}
\end{array}
$$

This formula can be verified immediately by differentiation with respect to $\theta$ which is very tedious. An intuitive argument for the correctness of eq. (8) will be provided in Section 4 where we demonstrate that it coincides in the special case of the price of a single switch option with the result derived independently by using a static replication argument. The reader interested in a direct proof of eq. (8) by integration may be referred to the similar proof of Theorem 3.1 in Pechtl (1997).

In the following we consider the payout profile $\Phi_{2}=\Phi_{2}\left(\lambda_{1}, \lambda_{2}, T, K ; S\right)$ of a dual switch option with a total lifetime $T_{1}$ from $t=-T_{0}$ to $t=T$, i.e. $T_{1}=T_{0}+T$, at time $t=0$. In the time-interval $\left[-T_{0} ; 0\right]$ the price-process $S$ respectively the no-arbitrage process $X$ is described by its historical data. Then $\Phi_{2}\left(\lambda_{1}, \lambda_{2}, T, K ; S\right)$ is determined by

$$
\Phi_{2}\left(\lambda_{1}, \lambda_{2}, T, K ; S\right)=\left\{\lambda_{1} \int_{t=-T_{0}}^{T} \mathbf{1}\left[S_{t}>K\right] d t-\lambda_{2} \int_{t=-T_{0}}^{T} \mathbf{1}\left[S_{t} \leq K\right] d t\right\}^{+} .
$$

¿From this we immediately obtain

$$
\Phi_{2}\left(\lambda_{1}, \lambda_{2}, T, K ; S\right)=\left\{\left(\lambda_{1}+\lambda_{2}\right) \Gamma_{+}\left(T_{1}, K\right)(S)-\lambda_{2} T_{1}\right\}^{+} .
$$


For $\lambda_{1}+\lambda_{2}=0$ and if $\lambda_{2}>0$ we trivially have $\Phi_{2}\left(\lambda_{1}, \lambda_{2}, T, K ; S\right)=0$, i.e. $\pi_{B S}\left(\Phi_{2}\left(\lambda_{1}, \lambda_{2}, T, K ; S\right)\right)=0$, if $\lambda_{2} \leq 0$ we have $\Phi_{2}\left(\lambda_{1}, \lambda_{2}, T, K ; S\right)=-\lambda_{2} T_{1}$, i.e. $\pi_{B S}\left(\Phi_{2}\left(\lambda_{1}, \lambda_{2}, T, K ; S\right)\right)=-r^{-T} \lambda_{2} T_{1}$, respectively. Thus for further discussion we only take the case $\lambda_{1}+\lambda_{2} \neq 0$ into consideration.

Let now be $\gamma_{0}=\Gamma_{+}\left(T_{0}, K\right)(S)=\int_{t=-T_{0}}^{0} \mathbf{1}\left[S_{t}>K\right] d t$ and $\tau_{0}=\frac{\lambda_{2}}{\lambda_{1}+\lambda_{2}} T_{1}-\gamma_{0}$.

Then we have for $\lambda_{1}+\lambda_{2}>0$

$$
\Phi_{2}\left(\lambda_{1}, \lambda_{2}, T, K ; S\right)=\left(\lambda_{1}+\lambda_{2}\right)\left\{\Gamma_{+}(T, K)(S)-\tau_{0}\right\} \mathbf{1}\left[\Gamma_{+}(T, K)(S)>\tau_{0}\right],
$$

respectively for $\lambda_{1}+\lambda_{2}<0$

$$
\Phi_{2}\left(\lambda_{1}, \lambda_{2}, T, K ; S\right)=\left(\lambda_{1}+\lambda_{2}\right)\left\{\Gamma_{+}(T, K)(S)-\tau_{0}\right\} \mathbf{1}\left[\Gamma_{+}(T, K)(S)<\tau_{0}\right] .
$$

We use those representations of $\Phi_{2}$ to calculate the corresponding prices in the Black \& Scholes model.

(i) For $\lambda_{1}+\lambda_{2}>0$ we obtain using eqs. (6) to (8)

$$
\begin{array}{r}
\pi_{B S}\left(\Phi_{2}\left(\lambda_{1}, \lambda_{2}, T, K ; S\right)\right)= \\
r^{-T} E\left(\lambda_{1}+\lambda_{2}\right)\left\{\Gamma_{+}(T, K)(X)-\tau_{0}\right\} \mathbf{1}\left[\Gamma_{+}(T, K)(X)>\tau_{0}\right] \\
=r^{-T}\left(\lambda_{1}+\lambda_{2}\right) \int_{t \in\left(\tau_{0} ; T\right]}\left(t-\tau_{0}\right) d \mathbf{F}(t) \\
=r^{-T}\left(\lambda_{1}+\lambda_{2}\right) \int_{t \in\left(\tau_{0} ; T\right]}(1-\mathbf{F}(t)) d t \\
=r^{-T}\left(\lambda_{1}+\lambda_{2}\right) \int_{t \in\left(\tau_{0} ; T\right]}\left\{1-F\left(T, t, \frac{1}{\sigma} \log \frac{K}{S_{0}} ; \frac{\mu_{B S}}{\sigma}\right)\right\} d t \mathbf{1}\left[K \leq S_{0}\right] \\
+r^{-T}\left(\lambda_{1}+\lambda_{2}\right) \int_{t \in\left[0 ; T-\tau_{0}\right)} F\left(T, t, \frac{1}{\sigma} \log \frac{S_{0}}{K} ;-\frac{\mu_{B S}}{\sigma}\right) d t \mathbf{1}\left[K>S_{0}\right] \\
=r^{-T}\left(\lambda_{1}+\lambda_{2}\right)\left[t-J\left(T, t, \frac{1}{\sigma} \log \frac{K}{S_{0}} ; \frac{\mu_{B S}}{\sigma}\right)\right]_{t=\tau_{0}}^{T} \mathbf{1}\left[K \leq S_{0}\right] \\
+r^{-T}\left(\lambda_{1}+\lambda_{2}\right) J\left(T, T-\tau_{0}, \frac{1}{\sigma} \log \frac{S_{0}}{K} ;-\frac{\mu_{B S}}{\sigma}\right) \mathbf{1}\left[K>S_{0}\right] .
\end{array}
$$

(ii) For $\lambda_{1}+\lambda_{2}<0$ we obtain analogously

$$
\begin{array}{r}
\pi_{B S}\left(\Phi_{2}\left(\lambda_{1}, \lambda_{2}, T, K ; S\right)\right)= \\
r^{-T} E\left(\lambda_{1}+\lambda_{2}\right)\left\{\Gamma_{+}(T, K)(X)-\tau_{0}\right\} \mathbf{1}\left[\Gamma_{+}(T, K)(X)<\tau_{0}\right] \\
=r^{-T}\left(\lambda_{1}+\lambda_{2}\right) \int_{t \in\left[0 ; \tau_{0}\right)}\left(t-\tau_{0}\right) d \mathbf{F}(t) \\
=-r^{-T}\left(\lambda_{1}+\lambda_{2}\right) \int_{t \in\left[0 ; \tau_{0}\right)} \mathbf{F}(t) d t \\
=-r^{-T}\left(\lambda_{1}+\lambda_{2}\right) \int_{t \in\left[0 ; \tau_{0}\right)} F\left(T, t, \frac{1}{\sigma} \log \frac{K}{S_{0}} ; \frac{\mu_{B S}}{\sigma}\right) d t \mathbf{1}\left[K \leq S_{0}\right]
\end{array}
$$




$$
\begin{array}{r}
-r^{-T}\left(\lambda_{1}+\lambda_{2}\right) \int_{t \in\left(T-\tau_{0} ; T\right]}\left\{1-F\left(T, t, \frac{1}{\sigma} \log \frac{S_{0}}{K} ;-\frac{\mu_{B S}}{\sigma}\right)\right\} d t \mathbf{1}\left[K>S_{0}\right] \\
=-r^{-T}\left(\lambda_{1}+\lambda_{2}\right) J\left(T, \tau_{0}, \frac{1}{\sigma} \log \frac{K}{S_{0}} ; \frac{\mu_{B S}}{\sigma}\right) \mathbf{1}\left[K \leq S_{0}\right] \\
-r^{-T}\left(\lambda_{1}+\lambda_{2}\right)\left[t-J\left(T, t, \frac{1}{\sigma} \log \frac{S_{0}}{K} ;-\frac{\mu_{B S}}{\sigma}\right)\right]_{t=T-\tau_{0}}^{T} \mathbf{1}\left[K>S_{0}\right] .
\end{array}
$$

Concluding we obtain the main result of this paper, the Black \& Scholes formula for dual switch options.

Theorem 3.1. Let be $\Phi_{2}\left(\lambda_{1}, \lambda_{2}, T, K ; S\right)$ be the payout profile of a dual switch option as defined by eq. (9). Furthermore let $J(\tau, \theta, \kappa ; \mu)$ be defined by eq. (8). Then for $\lambda_{1}+\lambda_{2} \neq 0$ the Black 8 Scholes price of such an option is calculated by

$$
\begin{aligned}
\pi_{B S}\left(\Phi_{2}\left(\lambda_{1}, \lambda_{2}, T, K ; S\right)\right)= & r^{-T}\left|\lambda_{1}+\lambda_{2}\right|\left\{J\left(T, \theta\left(\tau_{0}, K ; S_{0}\right), \kappa\left(K ; S_{0}\right) ; \mu\left(K ; S_{0}\right)\right)\right. \\
& -\theta\left(\tau_{0}, K ; S_{0}\right) e\left(\lambda_{1}, \lambda_{2} ; K, S_{0}\right) \\
& \left.-\left(J\left(T, T, \kappa\left(K ; S_{0}\right) ; \mu\left(K ; S_{0}\right)\right)-T\right) e\left(\lambda_{1}, \lambda_{2}, K ; S_{0}\right)\right\},
\end{aligned}
$$

where

$$
\begin{array}{ll}
\tau_{0} & =\frac{\lambda_{2}}{\lambda_{1}+\lambda_{2}} T_{1}-\Gamma_{+}\left(T_{0}, K\right)(S), \\
\theta\left(\tau_{0}, K ; S_{0}\right) & =\tau_{0} \mathbf{1}\left[K \leq S_{0}\right]+\left(T-\tau_{0}\right) \mathbf{1}\left[K>S_{0}\right], \\
\kappa\left(K ; S_{0}\right) & =\frac{1}{\sigma} \log \frac{K}{S_{0}}\left\{\mathbf{1}\left[K \leq S_{0}\right]-\mathbf{1}\left[K>S_{0}\right]\right\}, \\
\mu\left(K ; S_{0}\right) & =\frac{\mu_{B S}}{\sigma}\left\{\mathbf{1}\left[K \leq S_{0}\right]-\mathbf{1}\left[K>S_{0}\right]\right\}, \\
e\left(\lambda_{1}, \lambda_{2}, K ; S_{0}\right) & =\mathbf{1}\left[\lambda_{1} \geq-\lambda_{2}\right] \mathbf{1}\left[K \leq S_{0}\right]+\mathbf{1}\left[\lambda_{1}<-\lambda_{2}\right] \mathbf{1}\left[K>S_{0}\right] .
\end{array}
$$

REMARK 3.2. The formula for $\pi_{B S}\left(\Phi_{2}\left(\lambda_{1}, \lambda_{2}, T, K ; S\right)\right)$ remains valid even for $\lambda_{1}^{(0)}+\lambda_{2}^{(0)}=0$ if the option's price is interpreted as limit of the pricing formula given in Theorem 3.1 for $\lambda_{1}+\lambda_{2} \rightarrow 0$ with $\lambda_{1}+\lambda_{2}>0$.

\section{APPLICATION OF THE RESULT TO SINGLE SWITCH OPTIONS}

An immediate consequence of Theorem 3.1 is the explicit formula for the Black \& Scholes price of a single switch option, i.e. an option with payout profile

$$
\Phi_{1}(\lambda, T, K ; S)=\lambda \int_{t=-T_{0}}^{T} \mathbf{1}\left[S_{t}>K\right] d t
$$

with total lifetime from $t=-T_{0}$ to $t=T$ and $\lambda>0$. Obviously this payout profile can be interpreted by $\Phi_{2}(\lambda, 0, T, K ; S)$ and we can easily provide the Black $\&$ Scholes price. With the notations of section 3 we have $\tau_{0}=-\gamma_{0}$ and we obtain 
(i) for $K \leq S_{0}$

$$
\pi_{B S}\left(\Phi_{1}(\lambda, T, K ; S)\right)=r^{-T} \lambda\left\{T+\gamma_{0}-J\left(T, T, \frac{1}{\sigma} \log \frac{K}{S_{0}} ; \frac{\mu_{B S}}{\sigma}\right)\right\},
$$

(ii) for $K>S_{0}$

$$
\begin{aligned}
\pi_{B S}\left(\Phi_{1}(\lambda, T, K ; S)\right) & =r^{-T} \lambda J\left(T, T+\gamma_{0}, \frac{1}{\sigma} \log \frac{S_{0}}{K} ;-\frac{\mu_{B S}}{\sigma}\right) \\
& =r^{-T} \lambda \gamma_{0}+r^{-T} \lambda J\left(T, T, \frac{1}{\sigma} \log \frac{S_{0}}{K} ;-\frac{\mu_{B S}}{\sigma}\right) .
\end{aligned}
$$

We conclude this result in the following corollary.

Corollary 4.1. The Black \& Scholes price $\pi_{B S}\left(\Phi_{1}(\lambda, T, K ; S)\right)$ of a single switch option with a total lifetime from $t=-T_{0}$ to $t=T$ and $\left[-T_{0} ; 0\right]$ already passed is calculated by

$$
\begin{aligned}
& \pi_{B S}\left(\Phi_{1}(\lambda, T, K ; S)\right)=r^{-T} \lambda \gamma_{0}+r^{-T} \lambda T \mathcal{N}\left(\frac{\log \frac{S_{0}}{K}}{\sigma \sqrt{T}}+\frac{\mu_{B S}}{\sigma} \sqrt{T}\right) \\
& +r^{-T} \lambda \cdot \operatorname{sign}\left(S_{0}-K\right)\left(-\frac{\log \frac{S_{0}}{K}}{\mu_{B S}}+\frac{\sigma^{2}}{2 \mu_{B S}^{2}}\right) \mathcal{N}\left(\operatorname{sign}\left(-\frac{\log \frac{S_{0}}{K}}{\sigma \sqrt{T}}-\frac{\mu_{B S}}{\sigma} \sqrt{T}\right)\right) \\
& -r^{-T} \lambda \cdot \operatorname{sign}\left(S_{0}-K\right) \frac{\sigma^{2}}{2 \mu_{B S}^{2}}\left(\frac{S_{0}}{K}\right)^{-2 \frac{\mu_{B S}}{\sigma^{2}}} \mathcal{N}\left(\operatorname{sign}\left(-\frac{\log \frac{S_{0}}{K}}{\sigma \sqrt{T}}+\frac{\mu_{B S}}{\sigma} \sqrt{T}\right)\right) \\
& +r^{-T} \lambda \frac{\sigma}{\mu_{B S}} \sqrt{\frac{T}{2 \pi}} \exp \left\{-\frac{1}{2}\left(\frac{\log \frac{S_{0}}{K}}{\sigma \sqrt{T}}+\frac{\mu_{B S}}{\sigma} \sqrt{T}\right)^{2}\right\},
\end{aligned}
$$

where $\lambda \gamma_{0}$ is the amount of payout determined by the historical path of $S$ in $\left[-T_{0} ; 0\right]$.

REMARK 4.2. The Black \& Scholes price formula for a single switch option can be also derived directly using the nice idea that the payout profile $\Phi_{1}(\lambda, T, K ; S)$ can be interpreted as a portfolio of simple digital options with increasing lifetimes $t$ and common strike $K$, where each of them pays out an amount of $\lambda d t$ or nothing at time $T$. Then by application of Fubini's theorem we obtain

$$
\begin{aligned}
\pi_{B S}\left(\Phi_{1}(\lambda, T, K ; S)\right) & =r^{-T} \lambda \gamma_{0}+r^{-T} \lambda E \int_{t=0}^{T} \mathbf{1}\left[X_{t}>K\right] d t \\
& =r^{-T} \lambda \gamma_{0}+r^{-T} \lambda \int_{t=0}^{T} \mathcal{N}\left(\frac{\log \frac{S_{0}}{K}}{\sigma \sqrt{t}}+\frac{\mu_{B S}}{\sigma} \sqrt{t}\right) d t .
\end{aligned}
$$

This elementary idea was presented without proof of the result in the framework of a general approach to classify derivative securities by static replication of options portfolios in Pechtl (1995). 
Now, the remaining integral can be calculated as follows. Integration by parts yields

$$
\begin{aligned}
& \int_{t=0}^{T} \mathcal{N}\left(\frac{\log \frac{S_{0}}{K}}{\sigma \sqrt{t}}+\frac{\mu_{B S}}{\sigma} \sqrt{t}\right) d t=T \mathcal{N}\left(\frac{\log \frac{S_{0}}{K}}{\sigma \sqrt{T}}+\frac{\mu_{B S}}{\sigma} \sqrt{T}\right) \\
& +\frac{1}{\sqrt{2 \pi}} \int_{t=0}^{T} \frac{1}{2}\left(\frac{\log \frac{S_{0}}{K}}{\sigma \sqrt{t}}-\frac{\mu_{B S}}{\sigma} \sqrt{t}\right) \exp \left\{-\frac{1}{2}\left(\frac{\log \frac{S_{0}}{K}}{\sigma \sqrt{t}}+\frac{\mu_{B S}}{\sigma} \sqrt{t}\right)^{2}\right\} d t .
\end{aligned}
$$

For further evaluation of the integral we suggest the following helpful Lemma 4.3.

Lemma 4.3. For $b, \nu \neq 0$ the following assertions hold.

$$
\begin{aligned}
& I_{1}(b, \nu, T):=\frac{1}{\sqrt{2 \pi}} \int_{t=0}^{T} \frac{1}{\sqrt{t^{3}}} \exp \left\{-\frac{1}{2}\left(\nu \sqrt{t}-\frac{b}{\sqrt{t}}\right)^{2}\right\} d t \\
& =\frac{1}{|b|}\left\{\mathcal{N}\left(\operatorname{sign}\left(\nu \sqrt{T}-\frac{b}{\sqrt{T}}\right)\right)+\right. \\
& \left.\exp \{2 \nu b\} \mathcal{N}\left(\operatorname{sign}\left(-\nu \sqrt{T}-\frac{b}{\sqrt{T}}\right)\right)\right\} \text {. } \\
& I_{2}(b, \nu, T):=\frac{1}{\sqrt{2 \pi}} \int_{t=0}^{T} \frac{1}{\sqrt{t}} \exp \left\{-\frac{1}{2}\left(\nu \sqrt{t}-\frac{b}{\sqrt{t}}\right)^{2}\right\} d t \\
& =\frac{\operatorname{sign}(b)}{\nu}\left\{\mathcal{N}\left(\operatorname{sign}\left(\nu \sqrt{T}-\frac{b}{\sqrt{T}}\right)\right)\right. \\
& \left.-\exp \{2 \nu b\} \mathcal{N}\left(\operatorname{sign}\left(-\nu \sqrt{T}-\frac{b}{\sqrt{T}}\right)\right)\right\} \text {. } \\
& I_{3}(b, \nu, T):=\frac{1}{\sqrt{2 \pi}} \int_{t=0}^{T} \sqrt{t} \exp \left\{-\frac{1}{2}\left(\nu \sqrt{t}-\frac{b}{\sqrt{t}}\right)^{2}\right\} d t \\
& =\frac{b \cdot \operatorname{sign}(b)}{\nu^{2}}\left\{\mathcal{N}\left(\operatorname{sign}\left(\nu \sqrt{T}-\frac{b}{\sqrt{T}}\right)\right)\right. \\
& \left.+\exp \{2 \nu b\} \mathcal{N}\left(\operatorname{sign}\left(-\nu \sqrt{T}-\frac{b}{\sqrt{T}}\right)\right)\right\} \\
& +\frac{\operatorname{sign}(b)}{\nu^{3}}\left\{\mathcal{N}\left(\operatorname{sign}\left(\nu \sqrt{T}-\frac{b}{\sqrt{T}}\right)\right)\right. \\
& \left.-\exp \{2 \nu b\} \mathcal{N}\left(\operatorname{sign}\left(-\nu \sqrt{T}-\frac{b}{\sqrt{T}}\right)\right)\right\} \\
& -\frac{2}{\nu^{2}} \sqrt{\frac{T}{2 \pi}} \exp \left\{-\frac{1}{2}\left(\nu \sqrt{T}-\frac{b}{\sqrt{T}}\right)^{2}\right\} \text {. }
\end{aligned}
$$


Proof. We consider the integrals $K_{1}(b, \nu, T)$ and $K_{2}(b, \nu, T)$ with

$$
\begin{aligned}
K_{1}(b, \nu, T): & =-\frac{1}{\sqrt{2 \pi}} \int_{t=0}^{T} \frac{1}{2}\left(\frac{\nu}{\sqrt{t}}+\frac{b}{\sqrt{t^{3}}}\right) \exp \left\{-\frac{1}{2}\left(\nu \sqrt{t}-\frac{b}{\sqrt{t}}\right)^{2}\right\} d t \\
& =\left[\mathcal{N}\left(\frac{b}{\sqrt{t}}-\nu \sqrt{t}\right)\right]_{t=0}^{T}, \\
K_{2}(b, \nu, T): & =\frac{1}{\sqrt{2 \pi}} \int_{t=0}^{T} \frac{1}{2}\left(\frac{\nu}{\sqrt{t}}-\frac{b}{\sqrt{t^{3}}}\right) \exp \left\{-\frac{1}{2}\left(\nu \sqrt{t}-\frac{b}{\sqrt{t}}\right)^{2}\right\} d t \\
& =\left[\exp \{2 \nu b\} \mathcal{N}\left(\frac{b}{\sqrt{t}}+\nu \sqrt{t}\right)\right]_{t=0}^{T} .
\end{aligned}
$$

Now we obtain eqs. (11) and (12) by

$$
\begin{gathered}
I_{1}=-\frac{1}{b}\left(K_{1}+K_{2}\right), \\
I_{2}=\frac{1}{\nu}\left(K_{2}-K_{1}\right) .
\end{gathered}
$$

Eq. (13) can be easily verified by

$$
I_{3}=\frac{1}{\nu}\left(b I_{2}-\frac{\partial}{\partial \nu} I_{2}\right) .
$$

REMARK 4.4. Since the payout profile $\Psi_{1}(\lambda, T, K ; S)$ of a single corridor option is defined by

$$
\begin{aligned}
\Psi_{1}(\lambda, T, K ; S) & =\lambda \int_{t=-T_{0}}^{T} \mathbf{1}\left[K_{1}<S_{t} \leq K_{2}\right] d t \\
& =\Phi_{1}\left(\lambda, T, K_{1} ; S\right)-\Phi_{1}(\lambda, T, K ; S)
\end{aligned}
$$

the Black \& Scholes price of such an option can be immediately obtained by Corollary 4.1.

\section{CONCLUSION}

In this paper an application of the distribution of occupation times of Brownian motion with drift to a certain type of range options is considered, in particular an explicit pricing formula for dual switch options is provided in the Black \& Scholes framework. Though the joint density of Brownian motion (with drift) at time $T$ and its occupation times up to $T$ is a familiar result of stochastic calculus [see Billingsley (1968)] it seems that an explicit version of the distribution of occupation times has not been provided as far as the author knows. However, the explicit knowledge of this distribution is helpful for the pricing of a large class of path-dependent options such as range or quantile options, especially with respect to a practical point of view. 


\section{References}

1. Akahori, J. (1995). "Some Formulae for a New Type of Path- Dependent Option." Ann. Appl. Probab. 5 383-388.

2. Billingsley, P. (1968). Convergence of Probability Measures. Wiley, New York.

3. Black, F. and Scholes, M. (1973). "The Pricing of Options and Corporate Liabilities." J. Polit. Econom. 81 637-659.

4. DAssios, A. (1995). "The Distribution of the Quantiles of a Brownian Motion with Drift and the Pricing of Path-Dependent Options." Ann. Appl. Probab. 5 389-398.

5. Duffie, D. (1988). Security Markets - Stochastic Models. Academic Press, New York.

6. Harrison, J. M. and Pliska, S. R. (1981). "Martingales and Stochastic Integrals in the Theory of Continuous Trading." Stochastic Process. Appl. 15 214-260.

7. Hart, I. and Ross, M. (1994). "Striking Continuity." RISK 7, № 6, 51-56.

8. Karatzas, I. and Shreve, S. E. (1988). Brownian Motion and Stochastic Calculus. Springer, Berlin.

9. MiurA, R. (1992). "A Note on Look-Back Options Based on Order Statistics." Hitotsubashi Journal of Commerce Management. 27 15-28.

10. Pechtl, A. (1995). "Classified Information." RISK 8, № 6, 59-61.

11. Pechte, A. (1997). "Distributions of Occupation Times of Brownian Motion with Drift." Unpublished manuscript. Deutsche Genossenschaftsbank Frankfurt am Main.

12. Yor, M. (1992). "On Some Exponential Functionals of Brownian Motion." Adv. in Appl. Probab. 24 509-531. 


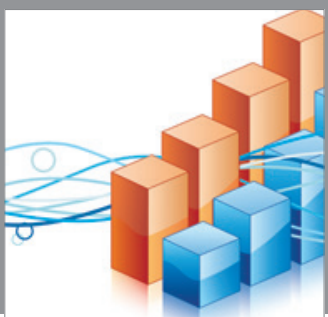

Advances in

Operations Research

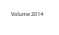

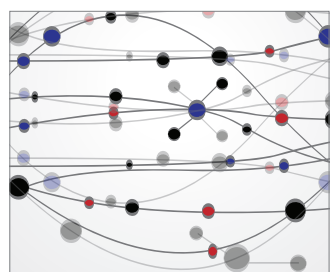

\section{The Scientific} World Journal
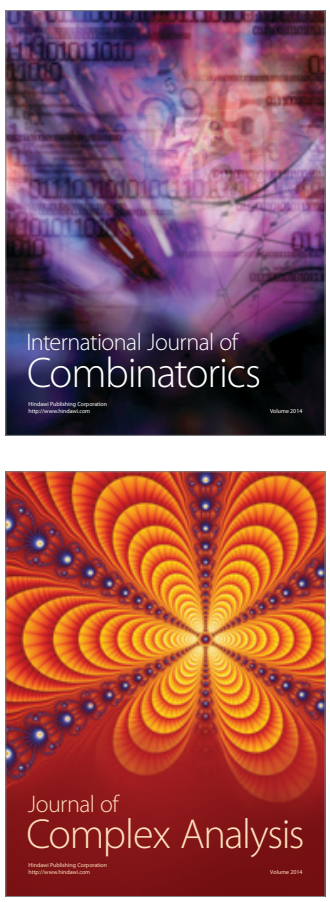

International Journal of

Mathematics and

Mathematical

Sciences
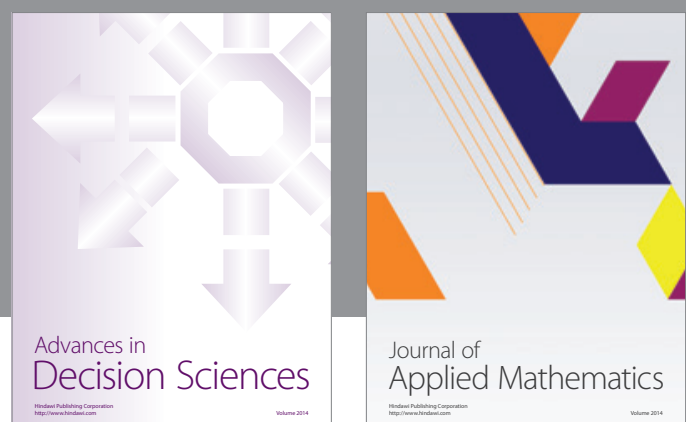

Journal of

Applied Mathematics
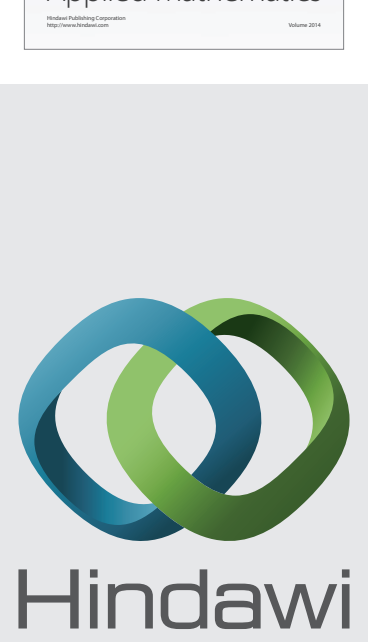

Submit your manuscripts at http://www.hindawi.com
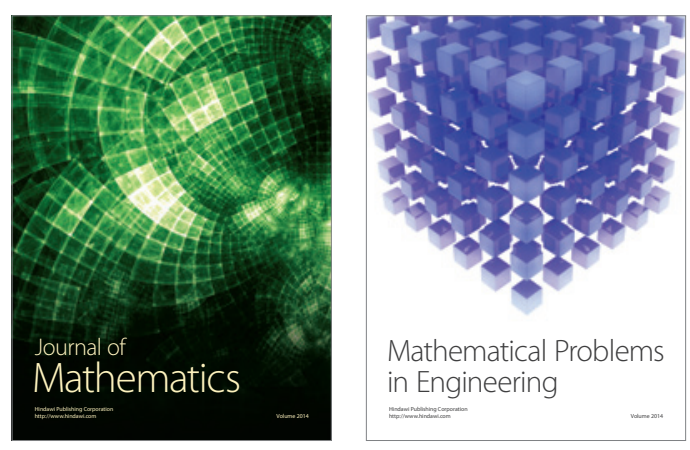

Mathematical Problems in Engineering
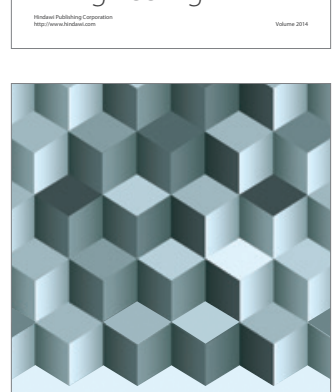

Journal of

Function Spaces
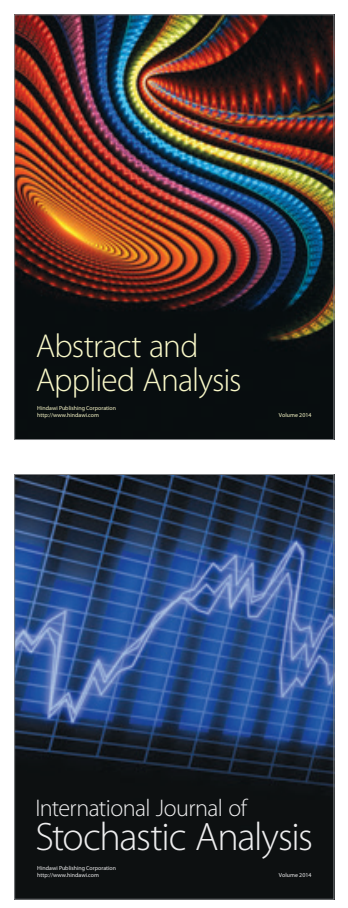

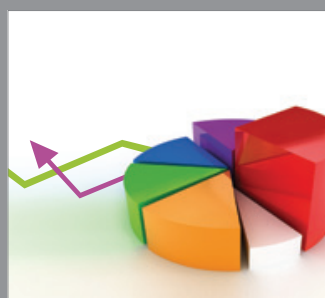

ournal of

Probability and Statistics

Promensencen
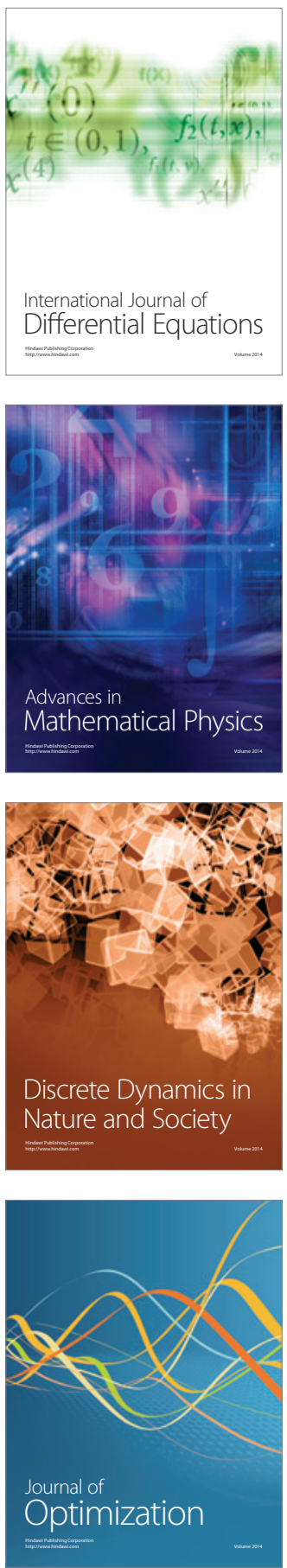